\title{
KERATOMALACIA IN SOUTHERN INDIA
}

BY

Major Robert E. Wright, I.M.S.

MADRAS

OF recent years a considerable amount of attention has been drawn to deficiency diseases. Various accessory food factors have been proved to be essential to the animal organism, and experimental deprivation of these substances has given rise to recognizable pathogenic states. Amongst these the experimental condition known as keratomalacia or xerophthalmia has been shown to be due to a deficiency of fat-soluble A, but so far as can be judged from the literature it is still questionable whether the clinical condition described under these names is similarly produced.

It may be well in the first instance to refer to the nature of experimental keratomalacia.

$\mathrm{McCollum}^{(1)}$ and his co-workers proved that in the case of white rats, the specific result of a lack of fat-soluble $\mathrm{A}$ in the diet is xerophthalmia. It is usually developed about the fortieth to fiftieth day of deprivation. He states, " the eyes become swollen so badly that they are opened with difficulty or not at all. The cornea becomes inflamed, and unless the missing dietary essential is supplied blindness speedily results." He obtained complete recoveries within two weeks of starting butter fat 5 per cent. in the diet, provided the animals were not previously blind. Normal eyelids could be restored even if the cornea was destroyed.

Professor Hopkins ${ }^{(2)}$ describes keratomalacia or xerophthalmia as consisting primarily and essentially in an abnormality of the corneal tissue which exhibits an exceptionally low resistance to a specific deficiency. He says, " the onset is sudden and is first noticed as an opacity but the symptoms develop so rapidly that it is hard to follow their sequence," within a few days there is complete panophthalmitis. He draws attention to the facility with which the experimental condition may be stopped with a little cod liver oil.

Secondly, in order to view the subject in its relation to human food, it is necessary to consider the sources of fat-soluble A, the possible effects of diets deficient in this substance, the influence of other substances such as salts and protein in the diet, and the likelihood of destroying fat-soluble $\mathrm{A}$ in the preparation of articles for food.

One gathers from the perusal of McCollum's work that there is very little fat-soluble $\mathrm{A}$ in diets other than those which contain green leafy vegetables and fat of milk, and that the great sources of this dietary essential are milk, butter, eggs, green leafy vege- 
tables and glandular organs. Diets composed of seeds and their products, tubers, roots, meats, etc., in the amounts in which they are ordinarily consumed do not furnish enough of this substance for the maintenance of an optimum state of well being. They may, however, when other deficiencies are corrected, contain enough fat-soluble $A$ to induce fairly good growth and prevent xerophthalmia. Diets made up largely of substances poor in fatsoluble A do not supply enough of this substance to prevent xerophthalmia indefinitely, or under the stress of lowering conditions such as pregnancy. Such diets bring an animal into what McCollum calls the "twilight zone," where small shifts in the quality of the diet with respect to any factor may determine the pathogenic state. Another element to which he calls attention is the digestive disturbance caused by bad fare, which may beget xerophthalmia not due to deficiency in the diet, but to the impaired vitality. He makes the interesting observation that fat-soluble $\mathbf{A}$ may be reduced to a certain minimum without producing xerophthalmia, whereas the same intake of this substance will not prevent the characteristic eye trouble when the intake of protein is likewise sufficiently lowered. A vegetarian diet without supplementary calcium, sodium, chlorine, will give rise to stunted growth, but a diet of cereals satisfactorily supplemented by salts of these elements and a protein of good quality, may for a long period prevent the onset of disease. The influence of salts in general, and those of calcium and sodium in particular is of great importance in association with border line dietaries, although their direct influence in keratomalacia is not indicated. In this connection Sir James Barr ${ }^{(2)}$ directed attention to the inorganic constituents of the blood, and the rôle of calcium and other salts in maintaining physiological equilibrium. He appeared to think that in the modern hue and cry after vitamines, the great importance of the inorganic constituents of the diet was overshadowed, and he indicated that in the consideration of such a complicated process as that of human metabolism, the interdependency of all factors had to be reckoned with. With regard to the stability of fat-soluble A, drying and heat (as in ordinary cooking) do not appear to destroy it, but oxidisation easily alters its nutritional qualities.

Long before the experimental keratomalacia or xerophthalmia of animals was described, clinicians in many parts of the world had recorded pathogenic states of huma beings under these names. It may well be asked, what is the relation between the two, if any? The opinions of some experimental and clinical observers on this point are worth noting.

Professor Gowland Hopkins ${ }^{(2)}$ expresses the opinion that the above condition as met with in deficiency feeding may have no 
clinical counterpart. He observed that the experiences of Bloch in Denmark that it has were not generally accepted by clinicians. $\mathrm{He}$ considered that protection was given by a very smail supply of the special factor, and he assumed that if there is any possibility of a condition with a similar origin occurring in human beings, this fact might account for its rarity. McCollum ${ }^{(1)}$ observes that it is important to enquire whether or not this disease has ever occurred in man, and refers to Mori's description of xerophthalmia in children in a time of food shortage. He considers that Mori's cases should be looked upon as a "deficiency disease " not hitherto recognised in its proper relation to diet. He states, "it is not a fat starvation, but if it be the same condition which McCollum and Simonds have definitely shown to be readily relieved in its early stages by the administration of such food stuffs as contain liberal amounts of fat-soluble A, it would not be relieved by feeding with vegetable fats in any amount." He leads one to infer that it ought to be relieved by feeding with foods rich in fat-soluble A.

Hess and Unger ${ }^{(3)}$ are of opinion that the danger to infants of a diet deficient in fat-soluble $\mathrm{A}$ is slight, provided it includes sufficient calories and is otherwise complete. They considered that in spite of the fact that this vitamine is not widely distributed in nature, a disorder that may be termed " fat-soluble deficiency," marasmus, or xerophthalmia is hardly to be apprehended from a clinical standpoint. They point out the importance of other elements in the diet in controlling disease, and that fat-soluble A is not the controlling influence when considered alone. In their experimental work on children, health and vigour were maintained despite amounts of fat-soluble vitamine so small as rarely to be encountered in times of peace. Hess ${ }^{(4)}$ failed to produce xerophthalmia in children deprived of fat-soluble A for periods of eight and nine months, nor did they develop rickets. He concluded from the experimental feeding of children that dangers from a lack of this dietary factor need not be apprehended if the diet is otherwise complete.

McCarrison(2) remarks on the frequency of the affection in Madras where he had seen a number of cases under the care of Lieut.-Colonel Kirkpatrick, once Superintendent of the Government Ophthalmic Hospital. Bloch ${ }^{(5)}$ described necrosis of the cornea with ulceration in children in whom recoveries followed feeding on breast milk or whole milk and cod liver oil.

Czerny and $\operatorname{Keller}^{(6)}$ describe a condition of the eyes in children suffering from malnutrition as a result of being restricted to a cereal diet.

Consideration of the various opinions recorded gives one the impression that there is a certain amount of confusion as to the 
relation between the keratomalacia of man and that of animals, and also between the different conditions described under this title in man. The term is perhaps a little unfortunate as it tends to limit one's attention to the eye. A widely circulated standard description of the human condition known as keratomalacia is that given in Fuchs' Text-book of Ophthalmology, 6th edition. There are numerous other records which need not be referred to here, but in order to avoid any misconception as to what the term keratomalacia implies when used with reference to cases in the Ophthalmic Hospital, Madras, a somewhat lengthy presentation of the subject is given below.

To begin with, Colonel Elliot, in his recent text book $^{(7)}$, under the heading " Night-blindness," makes it quite clear that the corneal trouble is only one of the manifestations of a great general disorder of a nutritional type. In the title to the chapter he draws attention to another marked symptom of the same disorder. He not only deals with the condition as observed by himself, but gives a very extensive reference to literature on the subject, so as to make it possible for comparison to be drawn between the conditions of this type occurring in other countries.

Colonel Kirkpatrick has furnished me with the following note on the subject, embodying his ideas not only of the clinical features, but also of the treatment, which I give in extenso :- " This condition occurs with considerable frequency in the South of India, and it is rare for the children's ward of the Madras Government Ophthalmic Hospital to be without some examples of the condition. It is seen most often during the periods of scarcity and famine, when all diseases due to lack of nutrition are common. Children under the age of five are most commonly affected, but it may be seen in older children and even in adults, especially those who are suffering from chronic inflammatory diseases of the intestine. Affected children have usually been bottle-fed on unsuitable food, or breast-fed by mothers whose nutrition is in a low state. It is quite common for Indian children in the South to be suckled until three or four years old (unless the arrival of another infant cuts off the available supply), and this appears to affect them deleteriously and to predispose to the disease. Keratomalacia may also be brought about in young children by the exhaustion which follows any acute disease. The principal factor in the causation of the disease appears to be the lack of some essential substances in the dietary, or, if such are present, to an inability to assimilate them. Experience has shown that these substances (or the most important of them) are present in cod liver oil, as its beneficial effect, if absorbed, is undoubted, but their exact nature is uncertain as yet. The 
characteristic feature of keratomalacia is a necrosis of the substantia propria of the cornea. This necrosis appears to be independent of a superficial microbic infection, and is not accompanied by any excessive vascular reaction. In many respects it - would appear to be a coagulation necrosis of the tissue. A personal impression (which has no value as evidence), has been formed that the thyroid gland and the liver are affected adversely by a food deficiency and that the changes in the cornea are largely the result of alteration in the secretions of these glands.

A premonitary sign of keratomalacia is the occurrence of a condition which closely resembles, and is probably identical with, the ordinary night-blindness. Some stiffening of the triangular area of conjunctiva on each side of the cornea is found. The area corresponds to the part of the bulbar conjunctiva which is exposed by the palpebral aperture and the base of the triangle is formed by the limbus. The changes are best marked on the temporal side. The disease affects both eyes. Even at this early stage the hair often appears dry and the surface of the skin roughened. The triangular patches are at first darker than the surrounding conjunctiva, but soon show a white formation like sea-foam or soaplather, on their surfaces. Some general darkening of the bulbar conjunctiva is generally present. As the disease progresses, the hair becomes more dry and scanty, and has a peculiarly bleached appearance; the skin also looks dry, rough and earthy. The pigmentation of the bulbar conjunctiva deepens and a faint grey haze appears between the centre and the periphery of the cornea, most commonly on the temporal side. No vascular reaction accompanies the appearance of this haze, which is situated in the substantia propria. The haze spreads and the whole cornea is soon involved. A well-marked icteric tinge is often now added to the pigmentation of the conjunctiva. The corneal surface next becomes dulled and the haze assumes a yellowish tint. A hypopyon may form at this stage. The tissues of the cornea now appear to melt away like thawing snow, and the entire structure yields en masse to the intraocular pressure and is cast off, so that the whole of the iris is exposed. The lens is next extruded and the vitreous lies bared in the pupil, giving the latter a jet-black appearance. A low type of inflammation is likely to set in now, or death, caused by the general condition, may take place. If the local condition is allowed to run its course, healing may sometimes occur and phthisis bulbi be the final result.

Cleanliness, warmth, and protection are the essentials in the local treatment of the disease. The conjunctival sac may be flushed out with warm normal saline solution twice or thrice daily and a drop of 10 per cent. argyrol or 0.75 per cent. nitrate of silver 
solution instilled. The conjunctiva should be kept constantly lubricated by frequent instillations of castor oil. Dry heat is often useful, and this may be applied by a bran bag (dry bran heated on a metal plate over hot coals or charcoal and then placed in a flannel bag), or a Japanese hand warmer; these are held over the eye in such a way as to exert no. pressure upon it. The hot air from an electric blower, if such an instrument is available, may be directed on the closed lids. Atropin is generally harmful, as it may excite conjunctival irritation, and it is unnecessary as iritis is nearly always absent. If it is considered advisable to dilate the pupil, hyoscin may be used with caution.

The constitutional treatment is the most important factor in staying the progress of the disease. If diarrhoea is present (as it generally is), a full dose of castor oil may be given; this is followed by the administration, every two or three hours, of an emulsion containing 10 to 20 minims of castor oil, according to the age of the patient, until the stools become yellow and the mucus disappears from them. Fair results were obtained by the use of treatment on these lines followed by a mixture which contained the chlorides of calcium, sodium, and ammonium. This mixture was prescribed in the earlier cases owing to an impression that calcium and the chlorides were deficient in the food, and it still appears to be of benefit. Cod liver oil is, however, the essential remedy for the disease ; it should be given with considerable care as the patient may not be able to digest it, and in such a case it should be rubbed into the skin of the axillae, back, and abdomen, but it should be administered by the mouth when it is at all possible to do so. There is often a scorbutic taint in this disease, and fruit juice should be given very freely. Whether caused by the intestinal disease or by the food deficiency, the thyroid gland is often atrophic and its secretion lacking; the use of thyroid extract is useful in such cases. Good frésh milk should be taken freely when the patient is able to to digest it. The disease is probably identical with the nightblindness accompanied by xerosis of the conjunctiva, which is so commonly seen amongst armies in the field and amongst others exposed to privations, and which is cured in its early stages by the use of liver in the diet. It is, however, a more advanced form of the condition, and, once it has been reached, the prognosis is much more serious."

At the risk of repetition, I might make a few further observations founded on cases seen locally. There is a certain amount of confusion between the condition described as epithelial xerosis and keratomalacia. No doubt in certain cases they tend to overlap, but there is not necessarily a triangular patch of xerosis nor yet white foam in keratomalacia, although such may occur. On the 
other hand, the triangular or rounded islands of greasiness on either side of the cornea may occur in an apparently otherwise normal individual, without night-blindness, and the term epithelial xerosis seems to fit such cases. The smokiness of the conjunctiva appears to be about the earliest sign in the eye. Here it may be observed that darkening and thickening of the conjunctiva has been noted as a symptom of scurvy by Neil McVicar ${ }^{(8)}$, in patients of all ages in South Africa. As distinct from the experimental condition the lids are not affected, except in so far as the palpebral conjunctiva is concerned, nor is there as a rule lacrymation or photophobia until the cornea is attacked. There is not necessarily any inflammatory reaction, and the vascularity of the conjunctiva is diminished rather than increased; quite a different picture from McCollum's experimental rats. There are certainly cases in which the cornea is infiltrated very early, with very limited conjunctival signs, but it seems to be rare for the cornea to be attacked first in humans, as it is in the experimental animals referred to by Professor Hopkins. The rapidity of the corneal destruction to which he refers is common in the clinical condition. The tears as a rule are scanty, and in very marasmic children are sometimes almost absent, but on the other hand they may be excessive in amount; in súch cases there is a wet sodden condition of the epithelium with some conjunctival swelling and hyperaemia, less smokiness, and a wet running nose, quite a contrast to the dry greasy variety with marked smokiness. In the only case investigated the lacrymal secretion was found to be a turbid, alkaline, albuminous fluid, with 1.75 per cent. total solids, and 0.73 per cent. chlorides (as $\mathrm{Na} \mathrm{Cl}$ )a decided diminution in the chlorides as compared with the normal. Night-blindness is by no means constant, but it is frequent. There appears to be a tendency to overlapping between the fundus pictures seen in this condition, retinitis punctata albescens, and retinitis pigmentosa. In the first there is perhaps only a pale fundus with possibly a fine white mottling; in the second there may be a pale retina, pale disc, with faintly red vessels thereon (indicative of a watery blood stream), and a mid-peripheral belt of discreet white spots; in the third there may be but a pale yellowish disc, with fine arteries and a light or normal coloured retinal ground with fine white mottling and perhaps an isolated line of pigment along a vessel. In all there may be night-blindness. Here it may be mentioned that the bone-corpuscle type of fundus in retinitis pigmentosa is not common in Madras.

In bad cases associated with wasting, the nasal, buccal and laryngeal mucous membranes are affected. The intestinal mucous membrane seems to be one of the most important seats of trouble, for whether the child, with greasy, smoky, concentrically rippled conjunctiva, be covered by a loose bag of skin, or fat and well 
nourished, there is generally a story of diarrhoea. In adults diarrhoea is the most common antecedent to a rapidly developing softening of the cornea. In children, broncho-prieumonia is sometimes an antecedent, and in severe cases frequently a terminal affection. A very important but inconstant feature is jaundice. Sometimes it is very marked and gives the scanty conjunctival débris in the fornix a greenish yellow colour. It appears to be more common in adults. It is sometimes associated with a small cirrhotic liver, at other times the liver is large. In one case a fairly well-to-do woman of middle age developed keratomalacia in association with an irregular, enlarged and painful liver, and intense jaundice, probably of a malignant nature.

Although the descriptions of experimental keratomalacia are somewhat meagre, there would appear to be considerable justification for the view that this clinical condition is not its counterpart. The latter is apparently much more complex, but may include such manifestations as are produced by deficiency feeding. In support of this view it may be stated that the rapid improvement produced in animals by feeding with substances rich in fat-soluble A has not been observed in human cases here.

With the idea of testing the value of introducing fat-soluble A together with protein into the diet, an experiment was undertaken in the second half of 1920 . A fresh mixture was prepared daily consisting of yolk of egg 1 part, fruit juice 1 , butter (ghee) 1 , raw meat juice 1. Adults received 8 ozs., babies 2 ozs. per day. Eight patients were treated with this mixture, in addition to their food for over a month; all improved, but not very rapidly, or any more markedly than eight similar cases treated with cod liver oil and triple chloride mixture, as recommended by Colonel Kirkpatrick.

Seven of these experimental cases had a history of fever or diarrhoea occurring before the onset of eye symptoms, one had no history of previous trouble. Numerous others (fourteen in each group) started the treatment, but as it was not kept up for over a month they are not included. This experiment took up a considerable time. It was personally supervised by Captain Craggs, who has had a large experience of such cases during his association with this hospital for a number of years. At times the mixture had to be suspended for a few days on account of digestive trouble, but on the whole the feeding was regularly carried out. Without going into tedious details of the experiment, it may be stated that the result was negative. It may be that the quantity of fat-soluble accessory factor in this diet was not sufficient. Apparently this is a matter of great importance, as has been emphasised by Harriette Chick and Elsie Dalyell(2) in connection with their Vienna work.

It would be impossible to give a correct impression of keratomalacia as seen here, without drawing attention to the population 
from which our cases are drawn, and the manner in which its individual members strive to exist on a minimum of food.

In Southern India the bulk of the poorer classes are always in, what McCollum terms, the " twilight zone." Not only is this so as regards fat-soluble $\mathrm{A}$, but also as regards other vitamines, and the optimum ratios of food principles in general. The majority of our cases of keratomalacia are noted as taking " ordinary diet," which consists of rice wetted with pepper water, or a thin vegetable curry with few, if any, green leafy vegetables in it. Milk, meat, eggs and butter, as a rule take no part in the diet, or possibly meat and milk may be partaken of occasionally. In certain districts fish may be partaken of fairly liberally, but this does not seem to protect, contrary to the experience of Mori in Japan, who noted that fish eaters escaped. When one considers that such is the state of affairs amongst the poorer classes in Madras, it is not surprising that there are many apparent evidences of deficiency and insufficiency with respect to diet. One has but to observe the average cases attending a large out-patient department in this part of the world, to have this impression suggested to one's mind. Take, for instance, the Government Ophthalmic Hospital. Not only does one see 60 to 100 cases of keratomalacia per annum; one also sees early senility, stunted growth, early cataract, arterial degeneration, atrophy of the skin, low body weight, slow cerebration, unaccountable oedemas, beri-beri, and a large variety of fundus conditions presumably dependent on vascular degeneration. From a general point of view the chief feature in the picture is loss of elastic tissues and general atrophy. Atrophy of the skin, subcutaneous tissues and muscles is the simplest expression of this almost universal deficiency. More complex pictures are produced as the result of structural alterations in the blood-vessels, and still more complex when the simpler forms of degeneration such as fatty change, or colliquative necrosis settle upon a particular tissue, for example, the cornea. In the two latter groups there are possibly other factors at work besides mere deficiencies of food constituents, and one has to think of toxaemias and endocrine alterations, either primary or as the result of deficiency in the first instance. The fact cannot be lost sight of, that there are at least two other important (and exceedingly common), conditions to be taken into account when considering this picture of extensive degeneracy: syphilis and consanguinity.

Assuming the necessity for a food deficiency in keratomalacia, it is theoretically possible that this may occur at different levels. First, the intake may be deficient, all other conditions being normal. Secondly, the diet may be adequate, but the absorbing surface may be defective. Thirdly, the diet may be adequate, the alimentary canal may be healthy, but the organ or organs which 
modify the products of digestion as absorbed from the blood stream may be at fault. As has been shown above there is ample opportunity for keratomalacia to occur at the first level amongst the poor in Southern India, but one would expect the disease to be more common if this were the only aetiological factor. As regards the second and third levels, it might appear at first sight that there are illustrative cases to be found. Numbers of our keratomalacia cases give a history of diarrhoea. What the exact nature of the disorder may be which is responsible it is hard to say, as no postmortem evidence is available, but it is a very important factor in the condition as it is the most frequent cause of death. It is not an essential feature as cases occur without affection of the alimentary tract. The fact that in certain cases adults develop typical eye symptoms late on in an intractable intestinal disorder, having previous to the onset of the enteritis been in good health, is not necessarily explained by the deprivation resulting from the defect in the alimentary canal. The enteritis may have merely determined the onset of the keratomalacia in an individual previously on a border line dietary. The same applies to those occasional cases in which the onset of liver disease in an apparently healthy individual determines the onset of typical eye symptoms. The theoretical consideration of the possibility, however, draws attention to the great importance of the alimentary tract and liver. Enough has been said about the former to emphasise the rôle of intestinal disease, but the liver deserves a little further attention.

There are certain clinical observations which suggest that the liver plays a very important part in keratomalacia, but it cannot be assumed that this is more than secondary in its relation to the disease.

That cod liver oil gives equal results to butter fat in about the same amounts, leads one to enquire whether there is not something of importance in this oil derived from the liver itself. Mori draws attention to the value of liver feeding, and his observations are supported by the custom, which has long been in vogue on the Malabar coast, of feeding children suffering from keratomalacia on fried liver.

I have it on very good authority that the results of such feeding are very beneficial. It may well be that feeding with other glandular organs would act in the same way, but there is no evidence that this is so unless the administration of thyroid extract be taken as such. With reference to the association of liver and eye disease, Oatman ${ }^{(9)}$ says, " hemeralopia, with and without pigmentation of the retina, has been observed in connection with chronic diseases of the liver." He also mentions that Landolt considered that an intimate relation existed between hepatic cirrhosis and diseases of the choroid and retina. 
Although many of our cases of keratomalacia have liver disturbance, it is more than probable that in the majority of such cases the liver is secondarily affected. Cirrhosis of the liver is frequent in Madras. In a recent series of 836 post-mortem examinations at the General Hospital, it was present in over 5 per cent. Chronic intestinal disease is also common here, and cirrhosis appears to have a definite relation to catarrhal and ulcerative affections of the intestine. Cunningham ${ }^{(10)}$ has called attention to the association of cirrhosis and jail dysentery in another part of India.

Since, as we have seen above, bad and insufficient food is almost universal amongst the poorer classes, we have locally all the factors necessary for the production of a vicious circle, such as one is accustomed to associate with keratomalacia, in which alimentary tract and liver play their part.

In conclusion the following summary may be given :-

There is in Southern India a clinical condition known by the name of keratomalacia, in which the symptoms of corneal degeneration form one of the leading features, inasmuch as it most frequently brings patients to seek relief; night-blindness is frequent but not so important a symptom.

The disease is not sharply marked off, but is a complex generalized disorder frequently associated with affections of the alimentary canal, grave constitutional disturbance, and marasmus.

In advanced cases it frequently ends fatally with terminal intestinal and lung affections. In less severe cases blindness is a common result.

It is practically always associated with deficiencies in the dietary of a general type, but general deficiency is so common, that to account for the comparative rarity of the disease one must suppose that other aetiological factors or determinants are necessary.

Clinical observations suggest the great importance of the liver, thyroid and intestine in these respects.

The condition does not appear to correspond to experimental keratomalacia or xerophthalmia, due to the deprivation of fatsoluble A, nor does it respond in the same way to the administration of a diet rich in this food essential.

It does, however, respond slowly to treatment, the chief measures being general care of the eyes and intestinal tract, and the administration of cod liver oil, liver, thyroid extract, and salts.

The prognosis as regards sight is bad if both corneae are attacked before treatment is commenced.

The condition is undoubtedly preventable, but the problem of its 
prevention, like so many of the great health problems of Southern India, largely resolves itself into an economic one to be dealt with by the financial rather than the medical authorities.

\section{REFERENCES}

1. McCollum.- " The newer knowledge of Nutrition."

2. Brit.Med. Jl., July 31, 1920.

3. Jl. of the Amer. Med. Assoc., January 24, 1920.

4. The Jl. of Biological Chemistry, Vol. XII, No. 3.

5. Ugeskruft für Laeger, Vol. LXXIX, p. 349, 1917.

6. Des Kindes, Leipsic, Part ii, p. 67, 1906.

7. Elliot.- "Tropical Ophthalmology."

8. South African Med. Record, August 14, 1920.

9. Oatman.- "Diagnosis of the Fundus Oculi." 1913 edition.

10. Indian Jl. of Med. Research, Vol. IV, No. 3. 1917.

\section{ANNOTATION}

\section{The Psychology of Vision}

The science of psychology, for science it may now be called since its rescue by Darwin from the mediaeval burdens imposed on it by metaphysical philosophy, is the most important science of all, for all others, even philosophy, are subject to its laws. Like all other sciences its laws must be based on observed facts and not on mere plausible hypotheses. Ophthalmology is in a very special way able to be of use in this connection, and it was a happy idea to devote a discussion at the last meeting of the Ophthalmological Society of the United Kingdom to the subject of the Psychology of Vision in Health and Disease. The discussion was introduced by papers by Prof. C. Spearman and Sir John Parsons. Both of these papers are already so condensed by two such masters of the art of condensation as to make further condensation impossible; they must be read in full. The subsequent discussion maintained the high scientific standard set. Sir Robert Armstrong-Jones laid stress on the importance of feeling tone, what has been described as the hedonic tone in dealing with the psychology of vision. He pointed out that in every sensation there is an attitude of the subject in the presence of an object, each sensation having its own state of feeling tone. He gave some interesting details on the effects of colour on the mental patients at Claybury and the Maudsley Hospital.

Dr. Kinnier Wilson dealt at considerable length with the psychological peculiarities in certain visual auras in epilepsy of 